\title{
Crawley-Boevey wird Humboldt-Professor in Bielefeld
}

Henning Krause

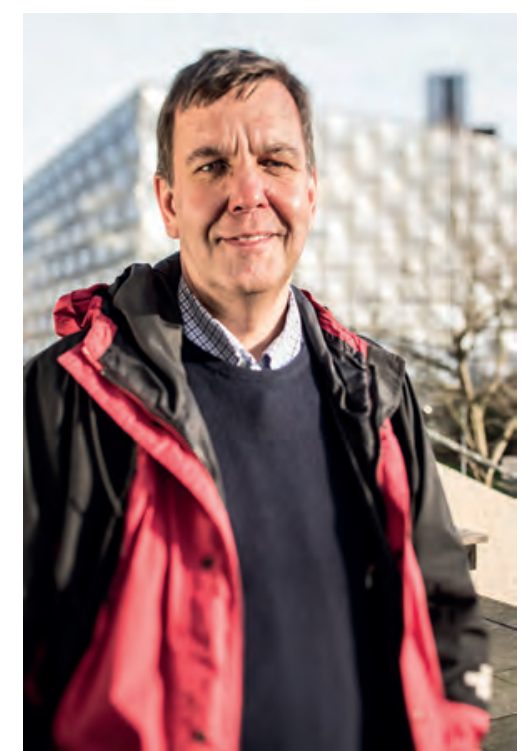

William Crawley-Boevey

(Foto: Humboldt-Stiftung/

Wolfgang Hemmann)

Im Oktober dieses Jahres wird der britische Mathematiker William Crawley-Boevey eine Alexander von Humboldt-Professur an der Universität Bielefeld antreten. Aus diesem Anlass sollen er und sein Arbeitsgebiet vorgestellt werden.

Seit 2009 vergibt die Alexander von Humboldt-Stiftung jährlich mehrere sogenannte Humboldt-Professuren, um internationale Spitzenforscher an deutsche Universitäten zu holen. Mit bis zu 5 Millionen Euro ist dies der höchstdotierte Forschungspreis Deutschlands. In vielen Fällen werden auf diese Weise deutsche Wissenschaftler aus dem Ausland zurückgewonnen. Bei Crawley-Boevey handelt es sich nun um einen Engländer, was auch die Frage nach der korrekten Aussprache aufwirft.

\section{William Crawley-Boevey ['wıljəm 'krəsli 'bu:vi]}

Geboren 1960 und ausgebildet an der Universität von Cambridge hat sich Crawley-Boevey erst nach seiner Promotion im Jahr 1985 seinem heutigen Arbeitsgebiet zugewandt. Dies geschah in Liverpool als Forschungsassistent bei Sheila Brenner und Michael Butler. Dort lernte er aus erster Hand die Darstellungstheorie endlichdimensionaler Algebren kennen. Der Darstellungstyp einer Algebra, genauer die sogenannte Zahm-Wild-Dichotomie war das Thema seiner ersten bedeutenden Veröffentlichung in diesem Gebiet [I]; sie brachte ihm sofort internationale Anerkennung. Claus Michael Ringel holte ihn daraufhin 1988 für knapp zwei Jahre an die Universität Bielefeld, und nach weiteren Jahren in Oxford wechselte CrawleyBoevey 1994 nach Leeds, wo er bis heute als Professor lehrt.

Darstellungstheorie von Algebren: Die Anfänge im SFB ,Theoretische Mathematik“ in Bonn

Um das Arbeitsgebiet von Crawley-Boevey auch innerhalb der deutschen Forschungslandschaft einzuordnen, lohnt der Blick zurück auf den seinerzeit von Hirzebruch in Bonn etablierten Sonderforschungsbereich „Theoretische Mathematik“. In den Jahren 1970-1974 war dort der französische Mathematiker Pierre (bzw. Peter) Gabriel zu Gast. Dieser hatte sich im Umfeld von Grothendieck und Serre mit seiner Dissertation „Des catégories abéliennes“ (1960) einen Namen gemacht [2].

Bereits in dieser berühmten und vielzitierten Schrift über abelsche Kategorien hatte Gabriel die Bedeutung der Längenkategorien herausgearbeitet. Zur Erinnerung: Ein Objekt $X$ in einer abelschen Kategorie hat endliche Länge wenn es eine endliche Kompositionsreihe

$$
0=X_{0} \subseteq X_{1} \subseteq \ldots \subseteq X_{n}=X
$$

besitzt, d.h. die Faktoren $X_{i} / X_{i-1}$ sind irreduzible Objekte, besitzen also keine echten von Null verschiedenen Unterobjekte. Nach Definition hat jedes Objekt in einer Längenkategorie endliche Länge und lässt sich nach dem Satz von Krull-Remak-Schmidt im Wesentlichen eindeutig in eine endliche direkte Summe von unzerlegbaren Objekten zerlegen. In diesem kategoriellen Kontext beschäftigt sich die Darstellungstheorie zunächst mit der Frage nach der Klassifikation der unzerlegbaren Objekte.

\section{Köcherdarstellungen: Der Satz von Gabriel}

Die Darstellungstheorie von Gruppen und Algebren hatte gerade auch in Deutschland eine lange Tradition; man denkt hier sofort an Frobenius, Schur und Brauer [3]. Neu war seit den 1960er Jahren der systematische Gebrauch von kategoriellen und homologischen Methoden.

In einem Oberwolfach-Vortrag stellte Gabriel 1970 eine Klassifikation der Köcher von endlichem Darstellungstyp vor. Was heute als Satz von Gabriel bekannt ist, liest sich im Tagungsbericht so [4]: 


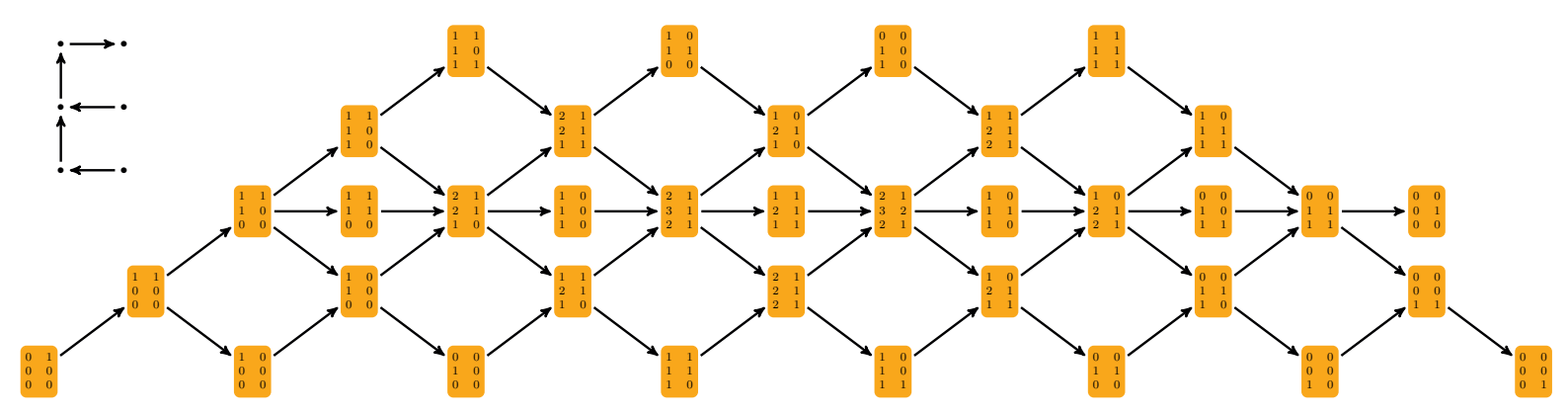

Abbildung I. Auslander-Reiten-Köcher der Darstellungen eines Köchers vom Dynkin-Typ $E_{6}$

P. Gabriel: Über einen Satz von Yoshii. Ein Graph $\Gamma$ besteht bekanntlich aus einer Punktmenge $E$, einer Pfeilmenge $K$ und aus zwei Abbildungen a, e: $K \rightrightarrows E$, die einem Pfeil die zugehörigen Anfangs- und Endpunkte zuordnen. Wir nehmen an, daß $K$ und $E$ endlich sind. Ersetzt man die Punkte des Graphen $\Gamma$ durch $k$-Vektorräume und die Pfeile durch $k$-lineare Abbildungen, so erhält man ein $k$-Diagramm vom Typ $\Gamma$. Es werden die Graphen beschrieben, die bis auf Isomorphie nur endlich viele direkt unzerlegbare Diagramme besitzen. Damit wird ein Satz von Yoshii (Osaka, 1956) neu hergeleitet und auch berichtigt.

Die besagten gerichteten Graphen sind übrigens genau die disjunkten Vereinigungen von Graphen vom DynkinTyp $A_{n}, D_{n}, E_{6}, E_{7}, E_{8}$ wie sie in der Lie-Theorie auftreten. Heute spricht man von Köchern statt Graphen, und die $k$-Diagramme sind deren $k$-lineare Darstellungen. Zum Beispiel gibt es für einen Köcher vom Dynkin-Typ $E_{6}$ bis auf Isomorphie genau 36 unzerlegbare Darstellungen - sie entsprechen bijektiv den positiven Wurzeln eines Wurzelsystems vom Typ $E_{6}$. Auf verschiedenste Weisen ergeben sich hier wunderschöne kombinatorische, geometrische und homologische Zusammenhänge zwischen Köcherdarstellungen und Lie-Theorie.

Die Kategorie der Darstellungen lässt sich mit Hilfe des sogenannten Auslander-Reiten-Köchers beschreiben: Die Eckpunkte entsprechen den unzerlegbaren Darstellungen und werden mit deren Dimensionsvektoren identifiziert; die Pfeile entsprechen den irreduziblen Morphismen (siehe die Abbildung).

\section{Unzerlegbare Darstellungen: Gabriels Bericht}

Veröffentlicht hat Gabriel diesen ersten Satz über Köcherdarstellungen in [5], aber den großen Zusammenhang skizziert er dann in einer bedeutenden Folgearbeit, in der er die damaligen Entwicklungen zusammenfasst und einander gegenüberstellt [6]. Sie betreffen in erster Linie die Algebren vom endlichen Darstellungstyp. Es werden also endlichdimensionale Algebren betrachtet mit der Eigenschaft, dass die Anzahl der Isomorphieklassen von unzerlegbaren Darstellungen endlich ist. Ohne im Detail auf die einzelnen Themen einzugehen, vermitteln die ersten Kapitelüberschriften einen guten Überblick:

I. The results of Dade-Janusz-Kupisch on modular representations of finite groups.

2. The results of Gelfand and Ponomarev on quadruples of vector spaces.

3. The results of Kleiner-Nazarova-Roiter on linear representations of ordered sets.

4. Quivers with only finitely many indecomposable representations.

5. The work of Roiter on the Brauer-Thrall conjectures.

6. The homological characterisation of M. Auslander.

Im Rückblick markiert diese Veröffentlichung den Aufbruch in eine neue Epoche der Darstellungstheorie, sowohl in Deutschland als auch international durch das Zusammenführen sehr verschiedener mathematischer Schulen.

Die erste internationale Tagung zur

Darstellungstheorie von Algebren

Das Interesse an den neuen Entwicklungen in der Darstellungstheorie führte $1974 \mathrm{zu}$ einer ersten internationalen Tagung. Sie wurde von Vlastimil Dlab mit Unterstützung von Peter Gabriel organisiert und fand in Ottawa statt. Das Poster dokumentiert die vielfältige Beteiligung. Damit wurde eine Tradition begründet. In der Regel gibt es nun alle zwei Jahre an wechselnden Orten eine solche internationale Tagung. In den Jahren 1998 und 2012 fand sie in Bielefeld statt.

Vom darstellungsendlichen Paradies zum zahmen Darstellungstyp

Zurück nach Bonn. Gabriel holte 1973 einen jungen Mathematiker an den dortigen Sonderforschungsbereich; 


\section{Carleton University Department of Mathematics}
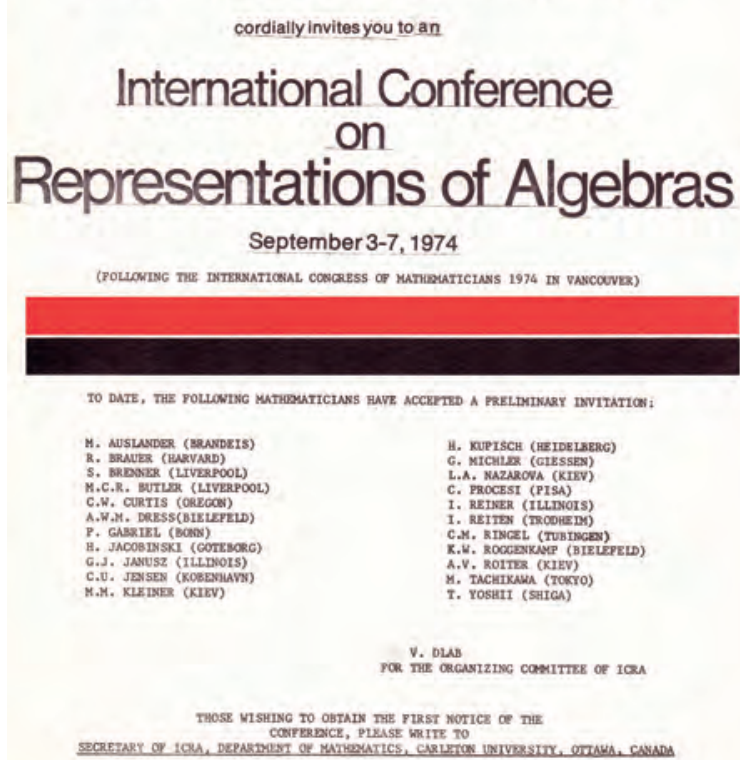

Poster der internationalen Tagung 1974 in Ottawa

dies war Claus Michael Ringel, der frisch habilitiert aus Tübingen kam.

In Bonn entstanden dann wichtige Arbeiten, die sich insbesondere mit Algebren vom zahmen Darstellungstyp befassten. Man spricht vom zahmen Typ, wenn sich die unzerlegbaren Darstellungen in jeder Dimension durch endlich viele 1-parametrige Familien beschreiben lassen.

Das Verständnis der zahmen Algebren war zunächst sehr rudimentär und eher von Beispielen geprägt. In Gabriels Bericht für den Internationalen Mathematikerkongress 1986 ist die Rede vom „darstellungsendlichen Paradies“ und er schreibt dann [7]: Leider bewohnen Algebren der Alltagspraxis meist die Hölle, wovon uns nur Randzonen bekannt sind mit ihren noch zahmen Wesen. Der 1984 von Ringel veröffentlichte wegweisende Band „,Tame algebras and integral quadratic forms“ verzichtet gänzlich auf eine Definition des zahmen Darstellungstyps [8]. Letztlich war es die anfangs erwähnte Arbeit von Crawley-Boevey über die Zahm-Wild-Dichotomie, die auf einem Ergebnis von Drozd basierend in Gabriels Hölle für klare Verhältnisse sorgte: In einem präzisen Sinne ist eine darstellungsunendliche Algebra entweder zahm oder wild, aber nicht beides zugleich.

\section{Von Bonn nach Bielefeld: Claus Michael Ringel}

Im Jahr 1978 folgte Ringel einem Ruf an die Universität Bielefeld, der er bis zu seiner Emeritierung 2010 treu blieb. Die Universität Bielefeld wurde so zu einem internationalen Zentrum der Darstellungstheorie von Algebren. Die Förderung durch die Deutsche Forschungsgemeinschaft hatte und hat daran einen wesentlichen Anteil: zwei Sonderforschungsbereiche (1989-2000 und 20052017) sowie zwei Schwerpunktprogramme (1984-1990 und 2009-2015) ermöglichten es immer wieder, exzellente Gäste und Nachwuchswissenschaftler nach Bielefeld zu holen. Auch die Alexander von Humboldt-Stiftung hat mit zahlreichen Stipendien und Forschungspreisen internationale Wissenschaftler an die Universität Bielefeld gebracht.

\section{Humboldt-Professur für Crawley-Boevey}

Nach der Emeritierung von Professor Ringel wird die Darstellungstheorie von Algebren in Bielefeld als Forschungsschwerpunkt mit vielfältigen Querbezügen fortgeführt, nicht zuletzt im Rahmen des derzeitigen Sonderforschungsbereichs „Spektrale Strukturen und topologische Methoden in der Mathematik". Die Alexander von Humboldt-Stiftung unterstützt diese Strategie mit der Einrichtung einer Humboldt-Professur, und William Crawley-Boevey wird zum Oktober 2016 dauerhaft von Leeds nach Bielefeld wechseln.

\section{Die Kac-Vermutungen}

Auch um einen Eindruck von den modernen Entwicklungen der Darstellungstheorie zu vermitteln, werden im Folgenden zwei der wichtigsten Forschungslinien von Crawley-Boevey vorgestellt.

Die erste dieser Forschungslinien betrifft die Kac-Vermutungen. In weitreichender Verallgemeinerung des grundlegenden Satzes von Gabriel in der Darstellungstheorie von Köchern hat Victor Kac um 1980 eine Untersuchung über unzerlegbare Darstellungen von Köchern und die Beziehungen zu Kac-Moody-Lie-Algebren veröffentlicht [9, 10]. Integraler Bestandteil sind Vermutungen über die sogenannten Kac-Polynome, die Isomorphieklassen unzerlegbarer Köcherdarstellungen über endlichen Körpern zählen, insbesondere eine Positivitätsvermutung über die Koeffizienten dieser Polynome sowie eine Konstante-Term-Vermutung, die einen direkten Zusammenhang zur Strukturtheorie von Kac-MoodyAlgebren vorhersagt.

Diese zunächst unscheinbar wirkenden Vermutungen sind von fundamentaler Bedeutung für das Langfristziel der Klassifikation unzerlegbarer Darstellungen von Köchern - die Positivität der Kac-Polynome wäre eine direkte Konsequenz aus einer, mehr erhofften als vermuteten, Klassifizierbarkeit der Darstellungen. Weiterhin lassen sich die Kac-Vermutungen in eine Reihe mit ähnlichen 
Vermutungen wie z. B. der Kazhdan-Lusztig-Vermutung stellen, denn ihre Untersuchung hat zu einer großen Menge an darstellungstheoretischen und algebraisch-geometrischen Fortschritten geführt.

Aufbauend auf seinen Arbeiten über präprojektive Algebren seit den späten 1990er Jahren hat William CrawleyBoevey 2004, in einer gemeinsamen Arbeit mit Michel Van den Bergh, die Kac-Vermutungen für sogenannte teilerfremde Dimensionsvektoren bewiesen; die KacPolynome werden dazu als Poincaré-Polynome der Kohomologie geeigneter Modulräume von Darstellungen präprojektiver Algebren interpretiert [II]. Diese Arbeit stellte den ersten Durchbruch zum Beweis der KacVermutungen seit ihrer Formulierung 1980 dar. Neben der fundamental neuen Idee, die Geometrie präprojektiver Algebren zu benutzen - die sicherlich aus dem tiefen Verständnis herrührt, das Crawley-Boevey für diese Klassen von Algebren entwickelt hatte - werden in dieser Arbeit vielerlei technische Schwierigkeiten aus der arithmetischen algebraischen Geometrie betreffend Reinheitseigenschaften der betrachteten Schemata überwunden.

In voller Allgemeinheit wurden die Kac-Vermutungen 2012 von Hausel, Letellier und Rodriguez-Villegas bewiesen [12]. Die Beweisstrategie fußt dabei im Wesentlichen auf der bereits genannten Arbeit von Crawley-Boevey und Van den Bergh. Sicherlich hat auch der persönliche Austausch von Crawley-Boevey mit Hausel eine wichtige Rolle gespielt.

\section{Das Deligne-Simpson-Problem}

Die zweite exemplarisch hier detaillierter diskutierte Forschungslinie betrifft das Deligne-Simpson-Problem. Ursprünglich aus der Theorie der gewöhnlichen Differentialgleichungen motiviert, besitzt dieses Problem eine rein algebraische Formulierung: Gegeben seien Matrizen in vorgegebenen Ähnlichkeitsklassen. In welchen Ähnlichkeitsklassen kann dann das Produkt bzw. die Summe der Matrizen liegen? Erstaunlicherweise gab es für dieses in den 1980er Jahren formulierte elementare Problem nur Antworten in einigen wenigen Spezialfällen!

Crawley-Boevey hat für die additive Variante, die nach der Summe der Matrizen fragt, eine vollständige Lösung, und für die wesentlich schwierigere multiplikative Variante eine fast vollständige Lösung angegeben. Über diese Lösungen berichtet sein Übersichtsartikel für den Internationalen Mathematikerkongress 2006 [13]. In beiden Fällen hat er das Problem in die Sprache von Darstellungen von Algebren übersetzt - für die additive Variante gelingt dies in der Sprache von Darstellungen von Köchern und präprojektiven Algebren, für die multiplikative Variante stellt sich die Sprache der parabolischen Vektorbündel auf projektiven Geraden bzw. logarithmischer Zusammenhänge als adäquat heraus [14, 15].
Als Anwendung mehrerer seiner Arbeiten über Modulräume von Darstellungen präprojektiver Algebren konnte Crawley-Boevey die additive Variante des Deligne-Simpson-Problems lösen [16]. In einer weiteren Serie von technisch und methodisch äußerst anspruchsvollen Arbeiten hat er dann die Theorie von Kac auf Kategorien parabolischer Vektorbündel übertragen, um ein analoges Resultat für die multiplikative Variante des Deligne-Simpson-Problems zu finden [17].

\section{Aufbruch aus dem darstellungsendlichen Paradies}

Einige der Entwicklungen seit Gabriels Bericht über unzerlegbare Darstellungen von 1973 wurden hier vorgestellt. Natürlich gab es viele weitere Fortschritte, von denen manche auch mit Bielefeld eng verknüpft sind; man denke nur an Kipptheorie, die systematische Untersuchung derivierter und stabiler Kategorien, oder an Ringel-Hall-Algebren.

Insgesamt zeigt der Rückblick und insbesondere das Guvre von Crawley-Boevey, dass es auch jenseits des darstellungsendlichen Paradieses erfreuliche Erkenntnisse und Strukturen gibt, die uns vor Höllenqualen bewahren und über die Darstellungstheorie hinaus von Nutzen sind.

\section{Dank}

Der Verfasser ist Markus Reineke für seine Mitwirkung zu besonderem Dank verpflichtet.

\section{Literatur}

[I] W. W. Crawley-Boevey, On tame algebras and bocses, Proc. London Math. Soc. (3) 56 (1988), no. 3, 45I-483.

[2] P. Gabriel, Des catégories abéliennes, Bull. Soc. Math. France 90 (1962), 323-448.

[3] C. W. Curtis, Pioneers of representation theory: Frobenius, Burnside, Schur, and Brauer, History of Mathematics, 15, Amer. Math. Soc., Providence, RI, 1999.

[4] Ringe, Moduln und homologische Methoden: 10.5. bis 16.5. 1970 / Kasch, Friedrich (Hrsg.); Rosenberg, Alex (Hrsg.): Math. Forschungsinst., 1970. - 15 S. (Tagungsbericht / Mathematisches Forschungsinstitut Oberwolfach; 1970, 16).

[5] P. Gabriel, Unzerlegbare Darstellungen. I, Manuscripta Math. 6 (1972), 7I-103; correction, ibid. 6 (1972), 309.

[6] P. Gabriel, Indecomposable representations. II, in Symposia Mathematica, Vol. XI (Convegno di Algebra Commutativa, INDAM, Rome, 1971), 81-104, Academic Press, London, 1973.

[7] P. Gabriel, Darstellungen endlichdimensionaler Algebren, in Proceedings of the International Congress of Mathematicians, Vol. I, 2 (Berkeley, Calif., 1986), 378-388, Amer. Math. Soc., Providence, RI, 1987.

[8] C. M. Ringel, Tame algebras and integral quadratic forms, Lecture Notes in Mathematics, 1099, Springer, Berlin, 1984.

[9] V. G. Kac, Infinite root systems, representations of graphs and invariant theory, Invent. Math. 56 (1980), no. I, 57-92. 
[10] V. G. Kac, Root systems, representations of quivers and invariant theory, in Invariant theory (Montecatini, 1982), 74-108, Lecture Notes in Math., 996, Springer, Berlin, 1982.

[II] W. Crawley-Boevey and M. Van den Bergh, Absolutely indecomposable representations and Kac-Moody Lie algebras, Invent. Math. I 55 (2004), no. 3, 537-559.

[12] T. Hausel, E. Letellier and F. Rodriguez-Villegas, Positivity for Kac polynomials and DT-invariants of quivers, Ann. of Math. (2) 177 (20I3), no. 3, I I47-I I 68.

[13] W. Crawley-Boevey, Quiver algebras, weighted projective lines, and the Deligne-Simpson problem, in International Congress of Mathematicians. Vol. II, I I7-129, Eur. Math. Soc., Zürich, 2006

[14] W. Crawley-Boevey, Geometry of the moment map for representations of quivers, Compositio Math. I26 (200I), no. 3, 257-293.

[15] W. Crawley-Boevey and P. Shaw, Multiplicative preprojective algebras, middle convolution and the Deligne-Simpson problem, Adv. Math. 20 I (2006), no. I, I80-208.
[16] W. Crawley-Boevey, On matrices in prescribed conjugacy classes with no common invariant subspace and sum zero, Duke Math. J. I I 8 (2003), no. 2, 339-352.

[17] W. Crawley-Boevey, Indecomposable parabolic bundles and the existence of matrices in prescribed conjugacy class closures with product equal to the identity, Publ. Math. Inst. Hautes Études Sci. No. 100 (2004), I7I-207.

Prof. Dr. Henning Krause, Fakultät für Mathematik, Universität Bielefeld, Universitätsstraße 25, 33615 Bielefeld hkrause@math.uni-bielefeld.de

Henning Krause (geb. 1962) studierte Mathematik in Berlin, Liverpool und Bielefeld, wo er 1991 promovierte. Sein Forschungsinteresse gilt der Darstellungstheorie von Algebren und der Homologischen Algebra. Nach Stationen in Leeds und Paderborn wurde er 2010 Nachfolger von Professor Claus Michael Ringel in Bielefeld.

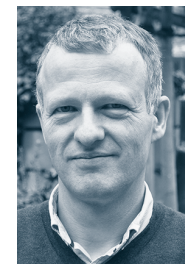

\section{Interview mit Friedrich Götze und Thomas Peternell}

Uta Deffke und Michael Joswig

Nach acht beziehungsweise elf Jahren im DFG-Fachkollegium für Mathematik sind Friedrich Götze (Universität Bielefeld, stellvertretender Sprecher des Fachkollegiums) und Thomas Peternell (Universität Bayreuth, Sprecher des Fachkollegiums) zum Mai 2016 ausgeschieden. Für die Mitteilungen wurden sie von Uta Deffke und Michael Joswig befragt.

Sie beide scheiden jetzt turnusgemäß aus dem Fachkollegium der DFG aus. Was waren dort Ihre Aufgaben?

TP: Das Fachkollegium Mathematik der DFG ist ein achtköpfiges Gremium, das innerhalb der DFG die Fachgruppe Mathematik vertritt. Wir geben Förderempfehlungen für Forschungsanträge, die bei der DFG eingehen. Das sind im Wesentlichen die Einzelanträge, die über das sogenannte Normalverfahren abgewickelt werden, also alles von der Sachbeihilfe über Forschungsstipendien, eigene Stellen bis hin zum Heisenberg- und Emmy-NoetherProgramm, inklusive der Forschergruppen.

Wie müssen wir uns die Arbeit des Fachkollegiums konkret vorstellen?

TP: Das Fachkollegium kommt viermal im Jahr zu seinen Beratungen zusammen, in Bonn oder in Berlin. Die Fachreferenten, Dr. Frank Kiefer und Dr. Carsten Balleier, be- kommen in jeder Runde um die vierzig bis fünfzig Anträge auf den Tisch und suchen passende Gutachter aus. Die schriftlichen Gutachten werden dann dem Fachkollegium vorgelegt.

FG: Dann diskutieren wir über die Anträge. Das kann natürlich auch sehr kontrovers sein. Schließlich sprechen wir eine Förderempfehlung aus, die wir dem Hauptausschuss vorlegen. In den allermeisten Fällen wird er dem auch folgen. Sollte es Zweifel geben, können auch noch weitere Gutachten eingeholt werden.

Wie viel Geld gibt es denn auf diese Weise zu verteilen?

TP: Der Jahresetat für das Normalverfahren lag in den letzten Jahren immer bei rund II,5 Millionen Euro, jetzt ist er auf 14 Millionen Euro gestiegen.

Ist vorgegeben, wie das Geld verteilt werden soll?

FG: Nein. Theoretisch könnten wir alles Geld in Forschergruppen investieren. Allerdings wäre dann die Reichweite begrenzt: Forschergruppen sind zwar ideal in der Mathematik, aber vergleichsweise teuer. Wir müssen aus einem Jahresetat gleich einen ganzen dreijährigen Block finanzieren, da kommen schnell zwei Millionen Euro zusammen. 